\title{
The Design and Thermal Reliability Analysis of a High-Efficiency K-Band MMIC Medium-Power Amplifier with Multiharmonic Matching
}

\author{
Y. Shang, ${ }^{1}$ H. Xu, ${ }^{1}$ J. Mo, ${ }^{1}$ Z. Wang, ${ }^{1}$ X. Xu, ${ }^{1}$ Z. Tu, ${ }^{1}$ X. Zhang, ${ }^{2}$ \\ H. Zheng, ${ }^{1}$ W. Chen, ${ }^{1}$ and F. Yu ${ }^{1}$ \\ ${ }^{1}$ School of Aeronautics and Astronautics, Zhejiang University, No. 38 Zheda Road, Hangzhou 310027, China \\ ${ }^{2}$ Hangzhou College of Commerce, Zhejiang Gongshang University, No. 18, Xuezheng Street, Hangzhou 310018, China
}

Correspondence should be addressed to J. Mo; jiongjiongmo@zju.edu.cn

Received 18 March 2016; Accepted 21 April 2016

Academic Editor: Gerard Ghibaudo

Copyright (c) 2016 Y. Shang et al. This is an open access article distributed under the Creative Commons Attribution License, which permits unrestricted use, distribution, and reproduction in any medium, provided the original work is properly cited.

A new high-efficiency K-band MMIC medium-power amplifier (PA) is designed with multiharmonic matching using GaAs pHEMT process technology. It has an operation frequency centered at $26 \mathrm{GHz}$ with a bandwidth of $2 \mathrm{GHz}$. A $20 \mathrm{dBm} 1 \mathrm{~dB}$ compression-point output power and $40 \%$ efficiency are achieved. A novel thermal reliability analysis method based on ICEPAK is proposed also to evaluate its thermal characteristic. The test result by using a QFI InfraScope ${ }^{\mathrm{TM}}$ infrared imaging system is compared with the simulation result. It agrees well with an accuracy within $\pm 1^{\circ} \mathrm{C}$ differences, which reflects the advantages of the thermal analysis method with respect to accuracy and convenience for use.

\section{Introduction}

GaAs monolithic microwave integrated circuit (MMIC) power amplifier (PA) design is a cutting-edge technology, which is widely used in the fields of communication [1-3], point-to-point network $[4,5]$, phased array radar system [6], and so forth. The trend of GaAs MMIC PA development is aiming at small size, high efficiency, and high reliability [7-10]. Especially, in the area of space application, due to the strict limitation of payload power supply and the high difficulty of payload repairing, the efficiency and reliability become two of the most important factors which have to be considered at the beginning of GaAs MMIC PA designs.

Recently, high-efficiency GaAs MMIC medium-power amplifier design has attracted increasing attentions. The authors in [11] have introduced a 4-stage broadband PA using GaAs pHEMT process with $24 \mathrm{dBm} 1 \mathrm{~dB}$-compression-point output power (P-1dB), $15 \%$ power added efficiency (PAE), and $3.36 \mathrm{~mm}^{2}$ chip size, over the frequency band from $17 \mathrm{GHz}$ to $26 \mathrm{GHz}$. A K-band driver amplifier and a K-band PA are introduced in [12]. The 6-stage power amplifier delivered
$23 \mathrm{dBm}$ output power from $17 \mathrm{GHz}$ to $36 \mathrm{GHz}$ with $8 \%$ PAE and $22.5 \mathrm{dBm}$ from $36 \mathrm{GHz}$ to $40 \mathrm{GHz}$ with $14 \%$ PAE. The 4 stage driver amplifier has achieved a $23.5 \mathrm{dBm}$ output power with $11 \%$ PAE from $18 \mathrm{GHz}$ to $35 \mathrm{GHz}$ and $22 \mathrm{dBm}$ output power with $8 \%$ PAE from $35 \mathrm{GHz}$ to $40 \mathrm{GHz}$. In [13], the authors have proposed a K-band broadband amplifier for local multipoint distribution system (LMDS) application. It has an operation frequency range from $24 \mathrm{GHz}$ to $28 \mathrm{GHz}$, $19.8 \mathrm{dBm}$ P-1dB, and $19.8 \%$ associated PAE with a chip size of less than $3.53 \mathrm{~mm}^{2}$. A K-band high-efficiency PA using $0.15 \mu \mathrm{m}$ GaAs pHEMT process technology is designed by the authors in [14]. The proposed PA has achieved $22 \mathrm{dBm}$ P$1 \mathrm{~dB}$ and $30 \%$ associated gain with a chip size of $1.5 \mathrm{~mm}^{2}$. In [15], the authors have demonstrated the design and test of a K-band GaAs MMIC which delivers a $22 \mathrm{dBm}$ P-1dB and 21\% PAE. In order to improve PA's efficiency, harmonic matching method is a key technique which has been used by many researchers. In [16], the authors have introduced a high-efficiency PA design using input and output harmonic terminations. The proposed PA has achieved an efficiency of $37 \%$ to $49 \%$ across its operation frequency band. A harmonic 


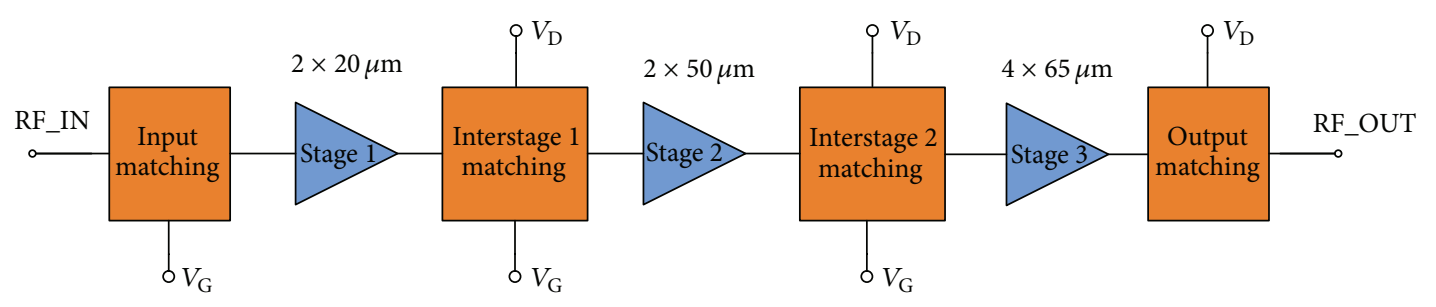

FIgURE 1: The block diagram of the proposed power amplifier.

matching method has been employed by the authors in [17] to design high-efficiency class-F MMIC PAs. The proposed PA has demonstrated an efficiency of $70 \%$ PAE for singleband $5.5 \mathrm{GHz} \mathrm{PA}, 58 \%$ PAE for $5 \mathrm{GHz} \mathrm{PA}$, and $51 \%$ PAE for $12 \mathrm{GHz}$ multiband PAs. In [18], the authors have proposed an ultrahigh-efficiency X-band class-E PA with broadband harmonic termination. The designed PA has demonstrated $49 \%-65 \%$ PAE over the frequency band of $9 \mathrm{GHz}-10 \mathrm{GHz}$ with $19 \mathrm{dBm}-22 \mathrm{dBm}$ output power.

In order to verify the reliability of the designed GaAs MMIC PAs, thermal analyses are performed. Many thermal simulation tools have been developed in the market. However, most of them are applied for general purpose [19]. Flotherm and ICEPAK are the tools for system-level thermal analysis [20-22]. On the other end of the spectrum, TCAD and APDT are most widely used for transistor-level thermal analysis, the user of which has to provide detailed information about the transistor down to the concentration of the semiconductor impurity [23-26]. Therefore, in order to estimate the thermal property of the designed MMIC PAs, a new method has to be proposed for accurate thermal simulation with less complexity and time consumption. Among the above simulation tools, ICEPAK is an advanced thermal analysis tool designed by ANASYS as its part of CFD suite. It is a great thermal analysis tool which has been widely used in electronics [20-22]. It has a great graphic user interface (GUI) and a comprehensive model library. With a suitable thermal model of GaAs MMIC PAs, it can be an ideal tool for thermal reliability analyses.

In this paper, a new high-efficiency K-band mediumpower amplifier using multiharmonic matching is introduced. It achieves a $20 \mathrm{dBm}$ P-1dB and a high PAE of $40 \%$ with a chip size of $2.64 \mathrm{~mm}^{2}$. In order to verify the thermal reliability, by using ICEPAK, an effective thermal model has been developed to simulate the entire GaAs MMIC PA at one go, with accuracy of less than $\pm 1^{\circ} \mathrm{C}$ temperature difference compared with the thermal image test using QFI InfroScope.

\section{PA Circuit Design}

The proposed PA is fabricated by using the $0.15 \mu \mathrm{m} \mathrm{GaAs}$ pHEMT technology. The typical parameters of the technology are illustrated in Table 1.

The designed circuit consists of three stages as illustrated in Figure 1 . The first stage is $2 \times 20 \mu \mathrm{m}$ pHEMT structure; the main purpose of this stage is to provide high linear gain for the whole circuit. The second stage is $2 \times 50 \mu \mathrm{m}$ pHEMT structure; it provides enough power to the input of the third
TABLE 1: Typical DC and RF characteristics of $0.15 \mu \mathrm{m}$ pHEMT process.

\begin{tabular}{lc}
\hline Parameter & Typical value \\
\hline Pinch-off voltage $\left(V_{\mathrm{PO}}\right)$ & $-1.3 \mathrm{~V}$ \\
Breakdown voltage $\left(V_{\mathrm{DG}}\right)$ & $16 \mathrm{~V}$ \\
Saturated drain current $\left(I_{\mathrm{dss}}\right)$ & $465 \mathrm{~mA} / \mathrm{mm}$ \\
Maximum drain current $\left(I_{\max }\right)$ & $620 \mathrm{~mA} / \mathrm{mm}$ \\
Peak transconductance $\left(g_{\mathrm{m}}\right)$ & $460 \mathrm{mS} / \mathrm{mm}$ \\
Transition frequency $\left(f_{\mathrm{t}}\right)$ & $90 \mathrm{GHz}$ \\
\hline
\end{tabular}

stage in order to drive the third stage into saturation region. The third stage is a $4 \times 65 \mu \mathrm{m}$ pHEMT structure and is the main part to deliver the required power to the circuit output. However, the third stage is the critical stage associated with the output power and efficiency of the designed PA. Therefore, in the following, the introduction is concentrated on the third stage only.

With respect to the third stage, the overall power input and output satisfy the following equation:

$$
P_{\mathrm{DC}}+P_{\text {in }}=P_{\text {diss }}+P_{\text {out }},
$$

where the total DC power is defined as $P_{\mathrm{DC}}=P_{\mathrm{DC}-\mathrm{G}}+P_{\mathrm{DC}-\mathrm{D}}=$ $V_{\mathrm{DS}} I_{\mathrm{D}}+V_{\mathrm{GS}} I_{\mathrm{G}}$ with $V_{\mathrm{DS}}$ and $I_{\mathrm{D}}$ being the drain source voltage and current of the third stage FET; $V_{\mathrm{GS}}$ and $I_{\mathrm{G}}$ are its gate voltage and current. $P_{\text {diss }}$ and $P_{\text {out }}$ are the power dissipated as heating and the total power delivered to the output port. The dissipated power and the total output power can be further written as in (2) with " $n$ " indicating the number of harmonics, " $\phi$ " is the angle difference between the voltage, and the current of the $n$th harmonic

$$
\begin{aligned}
& P_{\text {diss }}=\frac{1}{T} \int_{0}^{T} v_{\mathrm{DS}}(t) \cdot i_{\mathrm{D}}(t) d t, \\
& P_{\text {out }}=P_{\text {fund }}+P_{\text {harm }}=P_{\text {fund }}+\frac{1}{2} \sum_{n=2}^{\infty} V_{n} I_{n} \cos \left(\phi_{n}\right) .
\end{aligned}
$$

Therefore, in ideal case, when the dissipated power $P_{\text {diss }}$ and harmonic frequency power $P_{\text {harm }}$ are both zero, then the total output power of the PA equals the power of the fundamental frequency; the efficiency of the pHEMT reaches its maximum value of $100 \%$. The PAE of the pHEMT is defined as follows:

$$
\mathrm{PAE}=\frac{P_{\text {out }}-P_{\text {diss }}-P_{\text {in }}}{P_{\mathrm{DC}}} 100 \% .
$$




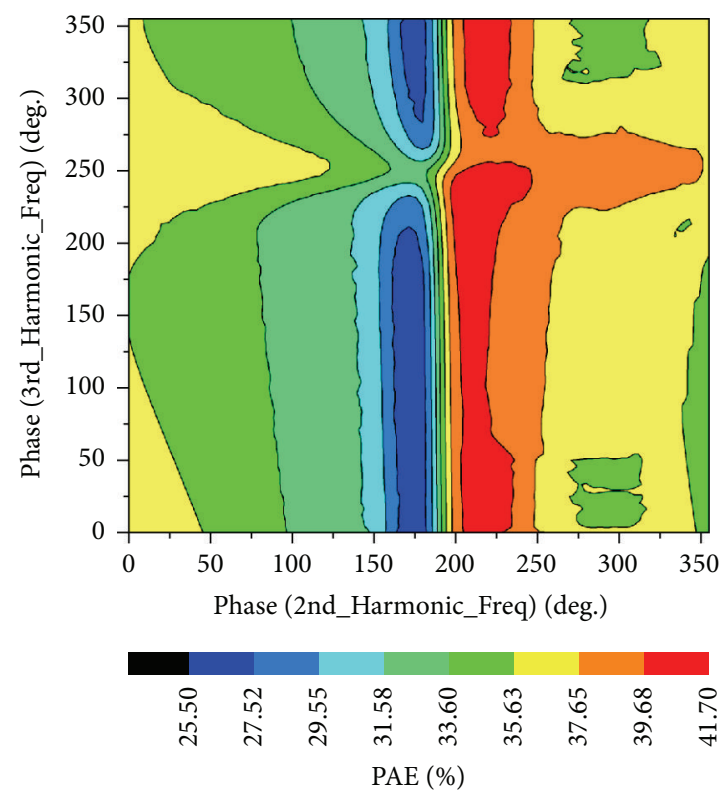

(a)

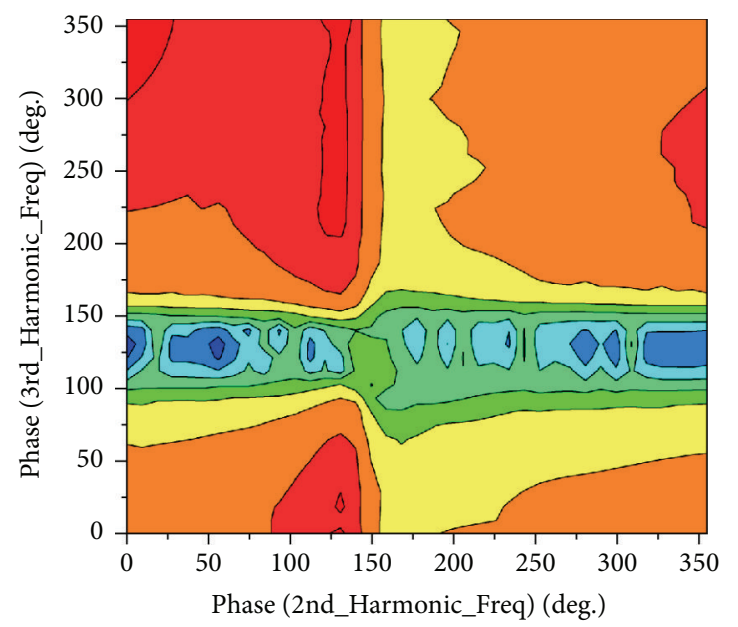

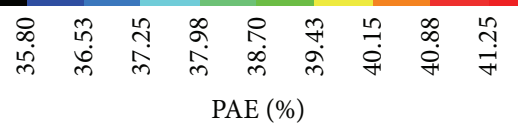

(b)

Figure 2: The source (a) and load (b) pull PAE contour of harmonics for the $4 \times 65 \mu \mathrm{m}$ pHEMT.

As illustrated in (3), when the harmonic frequency power is minimized, the total output power is maximized; it further leads to the minimization of dissipated power. The final PAE of the pHEMT is maximized. However, in most of the cases, only the second and third harmonics are significant and considered. With this in mind, the source-pull simulation of the pHEMT is illustrated in Figure 2. The horizontal axis is the phase of the second harmonic; the vertical axis is the phase of the third harmonic. The map indicates the PAE of the pHEMT at different harmonic phase grid. As demonstrated, the PAE changing is insensitive to the third harmonic phase change with only a slight discontinuity approximately between 250 degrees and 270 degrees. On the contrary, the PAE is very sensitive to the phase change of second harmonic; it reaches a maximum value of $41.7 \%$ at the phase sweeping between 205 degrees and 225 degrees. This result echoes the assumption made early that the higher harmonic is nonsignificant to the final harmonic power. It also indicates that only the second harmonic is worth mentioning during the input and output matching network design of the FET.

Figure 3 demonstrates the schematic circuit design of the per-matching network for the FET. It consists of two quarterwavelength shunt transmission lines TLa and TLc with respect to the second harmonic frequency, which eliminates the harmonic effect from the second and third stage outputs.

The transmission line and capacitor pair, TLb-C1 and TLd-C2, are for the purpose of input and output matching; they also have the use of tuning the harmonic frequency phase, especially for $\mathrm{C} 1$ and $\mathrm{C} 2$. The length of TLb and TLd can be arranged with respect to interconnection between two stages and the requirements of chip size. The photograph of the fabricated pHEMT MMIC power amplifier is illustrated in Figure 4 with chip size of $2.2 \mathrm{~mm} \times 1.2 \mathrm{~mm}$.

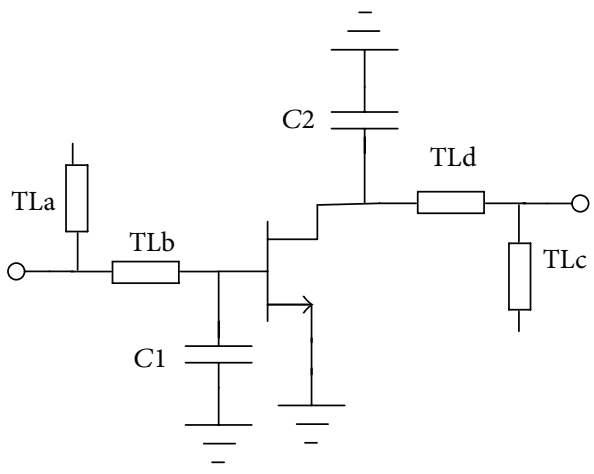

FIGURE 3: The multiharmonic prematching network for the $4 \times$ $65 \mu \mathrm{m}$ pHEMT cell.

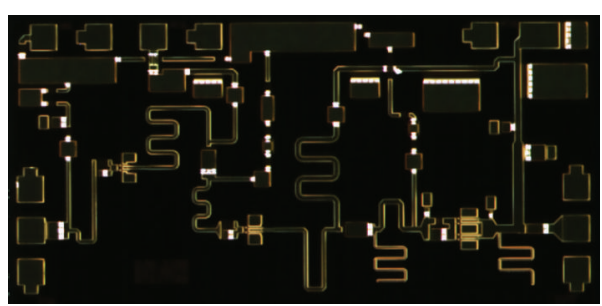

FIgure 4: Photograph of the fabricated pHEMT MMIC power amplifier.

The test results of the designed PA are done by using a network analyzer. The bias voltages of $\mathrm{VD}=5 \mathrm{~V}$ and $\mathrm{VG}=$ $-0.9 \mathrm{~V}$. The typical frequency response of the power amplifier is shown in Figure 5 along with the simulation results. Within its operation frequency band, the linear gain (S21) is greater than $25.5 \mathrm{~dB}$, and the input reflection is better than $15 \mathrm{~dB}$. 


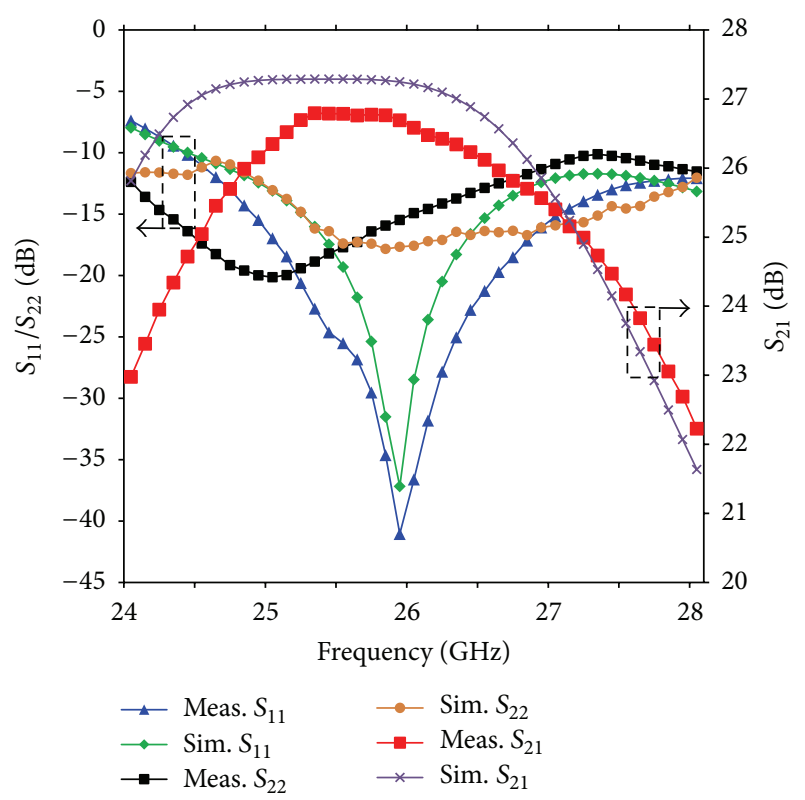

FIgURE 5: The simulated and measured $S$ parameter for the designed PA.

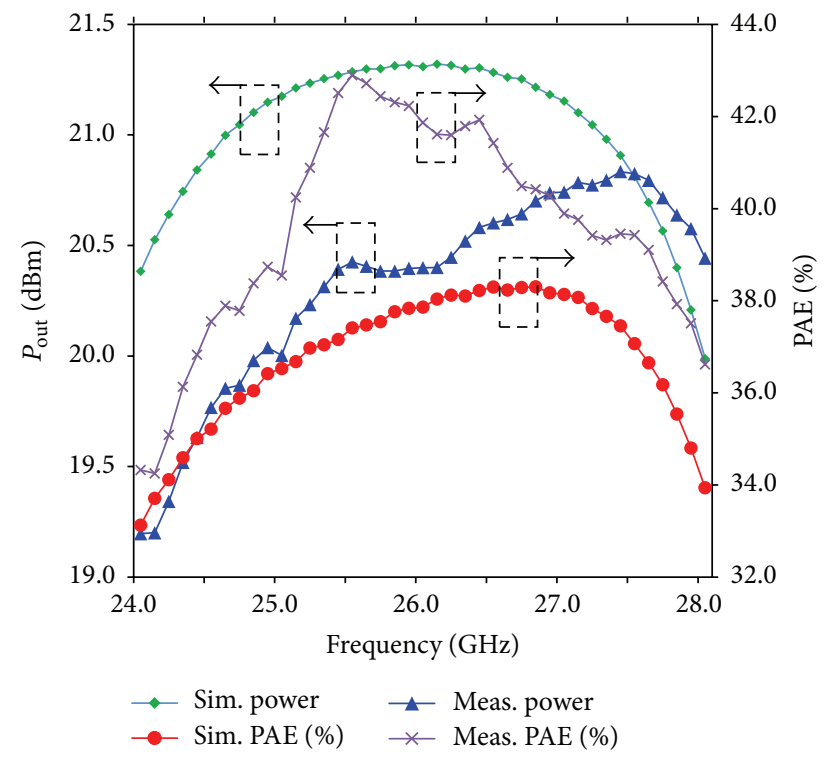

FIgURE 6: The simulated and measured P-1dB and PAE.

The output power and PAE are shown in Figure 6. As illustrated, the $\mathrm{P}-1 \mathrm{~dB}$ is $20 \mathrm{dBm}$, and the corresponding PAE is $40 \%$ across the entire operation frequency band form $25 \mathrm{GHz}$ to $27 \mathrm{GHz}$. However, the measured PAE is slightly better than the simulation result. On the other hand, the P-1dB is somewhat lesser than the simulated one. The reason can be that such change could be attributable to the variation of the process technology and the uncertainty of the test system.

\section{Thermal Analysis}

In order to evaluate the thermal characteristic of the designed PA, a thermal model is constructed in ICEPAK at the first

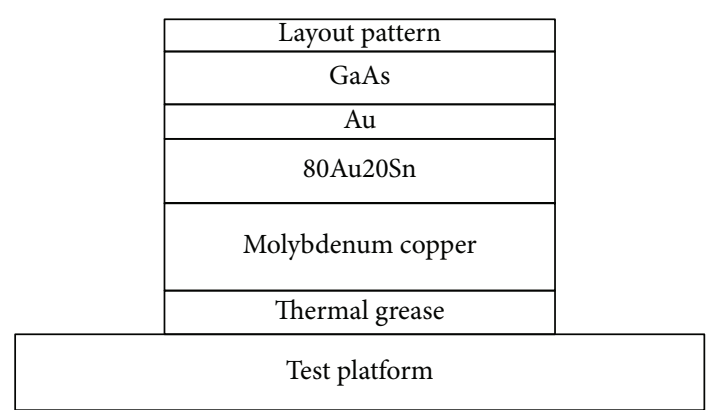

FIGURE 7: The schematic drawing of each layer for the simulation model.

instance. Herein, the overall model includes two parts: one is the designed PA and the other one is the heat sink on which the PA is mounted. A schematic drawing of each layer is illustrated in Figure 7.

The top three layers represent the MMIC PA circuit, which includes a layout pattern, GaAs epitaxial layer, and Au. The layout pattern is the main heat source which contains all the active circuit, transmission lines, and DC bias lines. The material for the layout pattern is all gold with thermal conductivity of $313 \mathrm{~W} / \mathrm{m} \cdot \mathrm{K}$. Then, an adhesive layer of solder material is used to mount the MMIC onto the top of molybdenum copper substrate. Finally, the PA and the substrate are fixed on a test platform. A layer of thermal grease is used to ensure the contact between the substrate and the heat sink. This model not only has a simple structure with respect to the simulation modeling but also can be generalized to different GaAs pHEMT based power amplifier design.

The simulation is done in two aspects. Figure 8 shows the case where only the three pHEMTs are simulated without any other information. The highest temperature is approximately $107.69^{\circ} \mathrm{C}$.

Figure 9 demonstrates the simulation result of the equivalent circuit of the designed PA. The hottest area is the third stage $\mathrm{pHEMT}$ with a temperature of $97.85^{\circ} \mathrm{C}$ which is responsible for delivering the requirement power to the output of the circuit. There is a $10^{\circ} \mathrm{C}$ difference from the previous section. This is due to that some of the heat has been dissipated from the pHEMT periphery such as the transmission line connected to the pHEMT and the vias connected to the back metal.

The thermal test of the designed PA using a QFI thermal imaging system InfraScope was performed to evaluate its thermal characteristic under full operation condition $\left(V_{\mathrm{DS}}=\right.$ $5 \mathrm{~V}, I_{\mathrm{DS}}=60 \mathrm{~mA}$ ) and assess the simulation method proposed. As illustrated in Figure 10, the thermal image of the third stage pHEMT has the highest temperature which is around $97.86^{\circ} \mathrm{C}$, only a small deviation from the simulation result. This indicates that the proposed thermal analysis is able to predict the thermal characteristic accurately.

\section{Discussion}

As stated in Section 2, the designed PA has achieved a PAE of $40 \%$ over its entire operation band with a minimum of 


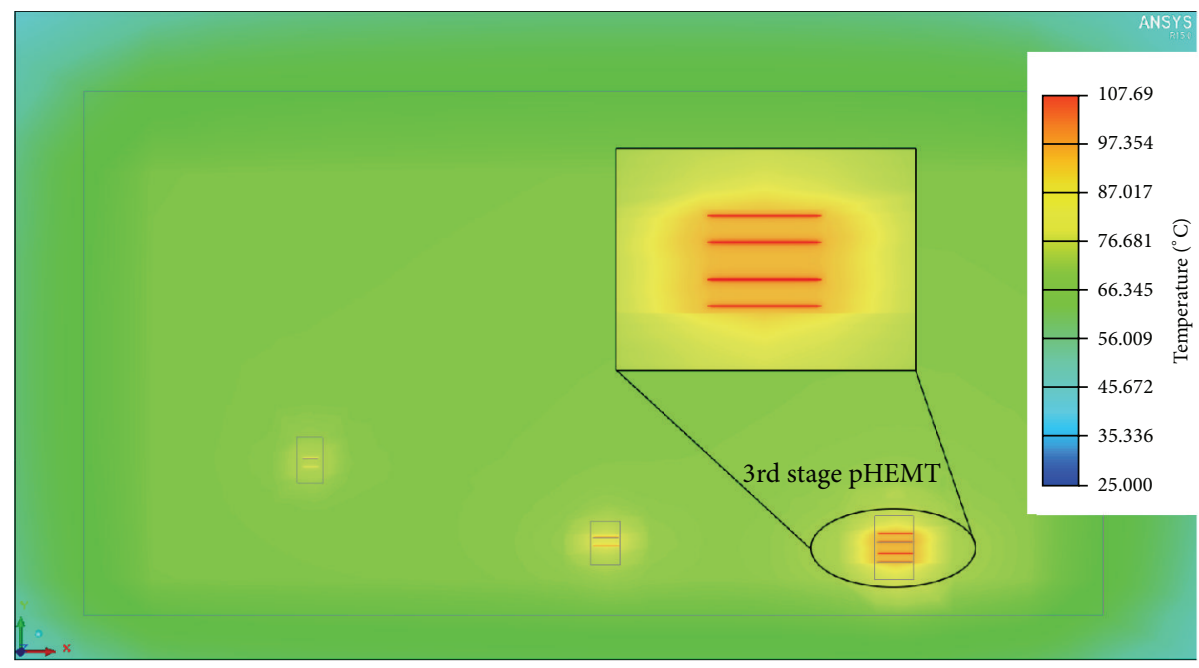

FIGURE 8: Thermal simulation of the PA with only three-pHEMT structure.

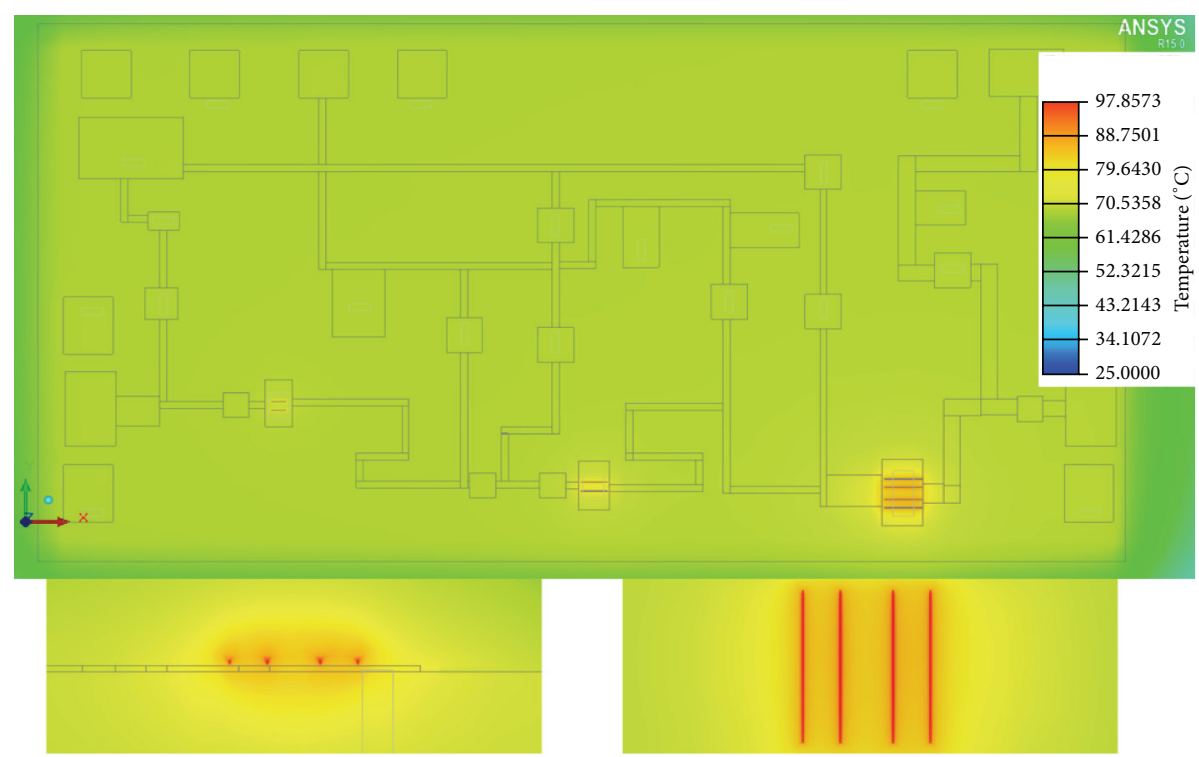

Figure 9: The simulation result of the designed PA using ICEPAK.

$20 \mathrm{dBm}$ P-1dB. The efficiency comparison of the K-band medium PAs and our work is illustrated in Table 2. From the efficiency point of view, our proposed PA achieved highest efficiency.

The thermal analysis is performed using a thermal model which is equivalent to the overall power amplifier circuit with ICEPAK software. The comparison of the simulation result and thermal imaging test result indicates that the method used herein is able to provide no more than $\pm 1^{\circ} \mathrm{C}$ accuracy estimation. The GUI (see Figure 11) of ICEPAK allows the user to build the equivalent simulation model more easily and fast. It also proves that it is not necessary to know the detailed information of the MMIC epitaxial layers and all the impurity concentrations. In case of thermal reliability design, it can accurately model the relative operation temperature of the designed MMIC by knowing the structure of the pHEMT and provide information such as the area of metal around transistor and the number of vias that are necessary during the MMIC design, especially in the case of high-powerdensity PA design. It also has the advantages of reducing the design cost due to chip fabrication and test and decreasing the design time cycling by reducing the design iteration used.

It also suggests that the thermal analysis can be done along with the PA's circuit design with a coarse drawing of the largest pHEMT cell, due to the highest power density occurring at those ranges. The fine simulation that can be at the stage of the overall circuit is almost certain stage to improve the design efficiency.

With respect to the proposed PA, it is applied to spaceborne phased array radar. One of the most troublesome reliability problems of the overall radar system is its PA, especially the thermal reliability of the proposed PA. With the same 
TABLE 2: The efficiency comparison of K-band PAs.

\begin{tabular}{|c|c|c|c|c|c|}
\hline Reference & Process/topology & Frequency & $P_{1 \mathrm{~dB}}$ & PAE & Chip size \\
\hline [11] & GaAs pHEMT/4 stages & $17-26 \mathrm{GHz}$ & $24 \mathrm{dBm}$ & $15 \%$ & $4.20 \mathrm{~mm} \times 1.80 \mathrm{~mm}$ \\
\hline \multirow{4}{*}{ [12] } & \multirow{2}{*}{ GaAs pHEMT/4 stages } & $18-35 \mathrm{GHz}$ & $23.5 \mathrm{dBm}$ & $11 \%$ & \multirow{2}{*}{$4.70 \mathrm{~mm} \times 1.90 \mathrm{~mm}$} \\
\hline & & $35-40 \mathrm{GHz}$ & $22 \mathrm{dBm}$ & $8 \%$ & \\
\hline & \multirow{2}{*}{ GaAs pHEMT/6 stages } & $17-36 \mathrm{GHz}$ & $23 \mathrm{dBm}$ & $8 \%$ & \multirow{2}{*}{ NA } \\
\hline & & $36-40 \mathrm{GHz}$ & $22.5 \mathrm{dBm}$ & $14 \%$ & \\
\hline$[13]$ & GaAs pHEMT/2 stages & $24-28 \mathrm{GHz}$ & $19.8 \mathrm{dBm}$ & $19.8 \%$ & $1.44 \mathrm{~mm} \times 2.45 \mathrm{~mm}$ \\
\hline [14] & GaAs pHEMT/4 stages & $17-35 \mathrm{GHz}$ & $22 \mathrm{dBm}$ & $30 \%$ & $1.50 \mathrm{~mm} \times 1.00 \mathrm{~mm}$ \\
\hline [15] & GaAs pHEMT/3 stages & $20-30 \mathrm{GHz}$ & $22 \mathrm{dBm}$ & $21 \%$ & $1.44 \mathrm{~mm} \times 2.45 \mathrm{~mm}$ \\
\hline This work & GaAs pHEMT/4 stages & $25-27 \mathrm{GHz}$ & $20 \mathrm{dBm}$ & $40 \%$ & $2.20 \mathrm{~mm} \times 1.20 \mathrm{~mm}$ \\
\hline
\end{tabular}

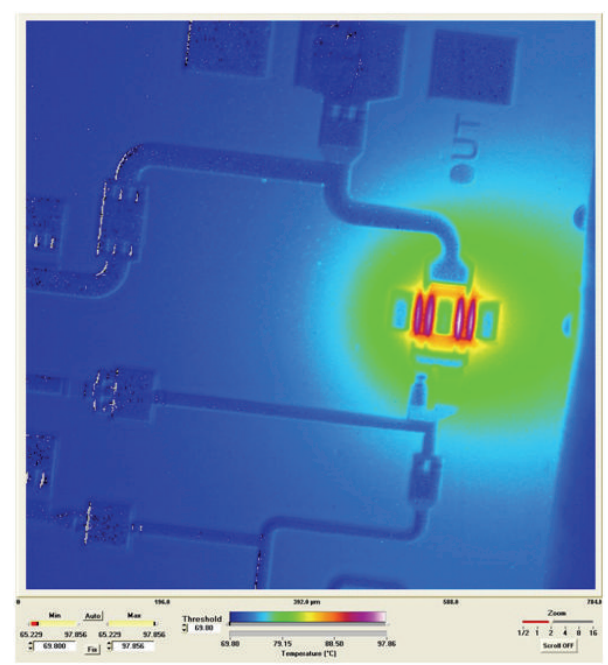

FIgURE 10: The infrared image of chip A under $V_{\mathrm{DS}}=5 \mathrm{~V}$ and $I_{\mathrm{DS}}=$ $60 \mathrm{~mA}$.

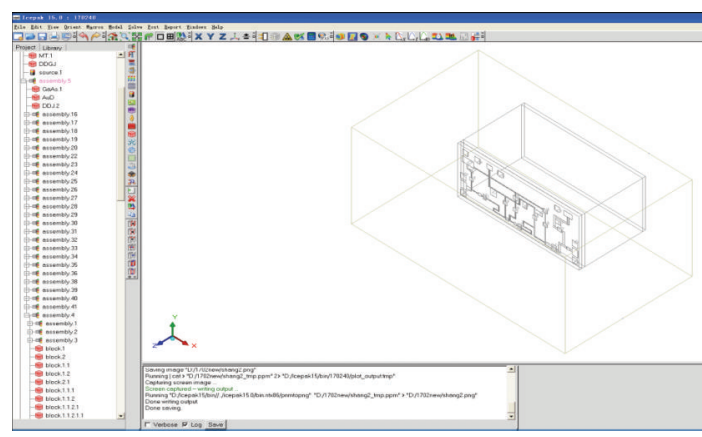

Figure 11: The example GUI window from ICEPAK.

output power, the higher output efficiency will lead to a lower dissipated power on its pHEMT and, further, lead to a longer device life time. Therefore, the thermal design is one of important design factors during the PA design. In this paper, the working temperature range for the proposed PA is designed between $-40^{\circ} \mathrm{C}$ and $85^{\circ} \mathrm{C}$. The final thermal test result has shown $97.86^{\circ} \mathrm{C}$ under normal bias condition at room temperature and the PAE at the same condition is not less than $40 \%$. Accordingly a widely used device junction temperature model is defined as $T_{j}=T_{\text {base }}+R_{\mathrm{th}} P_{\text {diss }}$ [27], which gives a proximate device junction temperature from $32.86^{\circ} \mathrm{C}$ to $157.86^{\circ} \mathrm{C}$, which is well below the normal GaAs pHEMT device junction temperature. This guarantees that the proposed device will have less chance to have thermal reliability problem during normal working condition. The aim of this analysis is achieved in this paper.

\section{Conclusion}

This paper has introduced the design of a K-band PA with multiharmonic matching. The final realized MMIC has a size of $2.64 \mathrm{~mm}^{2}, 20 \mathrm{dBm}$ P-1dB, and a high associated PAE of $40 \%$. A thermal analysis method is proposed for the verification of thermal reliability. By introducing the proposed equivalent thermal model in ICEPAK, accurate thermal characteristics of the designed MMIC PA have been achieved with less than $\pm 1^{\circ} \mathrm{C}$ of peak temperature difference comparing to the thermal image test result.

\section{Competing Interests}

The authors declare that they have no competing interests.

\section{Acknowledgments}

This work was supported by the National Science Foundation of China under Grant 61401395 and the Scientific Research Fund of Zhejiang Provincial Education Department under Grant Y201533913.

\section{References}

[1] Z. M. Tsai, K. Y. Lin, and H. Wang, "A 71-80 GHz medium power amplifier using 4-mil $0.15-\mu \mathrm{m}$ GaAs-PHEMT technology," in Proceedings of the Asia-Pacific Microwave Conference (APMC '11), pp. 1130-1133, 2011.

[2] A. Rasmi, A. Marzuki, M. A. Ismail, A. I. Abdul Rahim, M. R. Yahya, and A. F. A. Mat, " $0.5 \mu \mathrm{m}$ GaAs PHEMT medium power amplifier design using simple RC feedback amplifier for wireless LAN applications," in Proceedings of the Asia-Pacific Microwave Conference, pp. 1-4, December 2008. 
[3] C.-G. Yuan, S. M. J. Liu, D.-W. Tu et al., "0.15 micron gate 6-inch pHEMT technology by using I-line stepper," in CS MANTECH Conference, Tampa, Fla, USA, May 2009.

[4] S. Chen and S. Nayak, "A 1/2 Watt high linearity and wide bandwidth PHEMT driver amplifier MMIC for millimeterwave applications," in Proceedings of the IEEE MTT-S International Microwave Symposium Digest, pp. 1863-1866, IEEE, San Francisco, Calif, USA, 2006.

[5] V. Camarchia, S. Donati Guerrieri, G. Ghione et al., "A Kband GaAs MMIC doherty power amplifier for point-topoint microwave backhaul applications," in Proceedings of the International Workshop on Integrated Nonlinear Microwave and Millimetre-Wave Circuits (INMMiC '14), pp. 1-3, Leuven, Belgium, April 2014.

[6] A. A. Lane, J. A. Jenkins, C. R. Green, and F. A. Mayers, "S and C band GaAs multifunction MMICs for phased array radar," in Proceedings of the 11th Annual Gallium Arsenide Integrated Circuit (GaAs IC) Symposium, Technical Digest, pp. 259-262, San Diego, Calif, USA, October 1989.

[7] S.-J. Cho, C. W. Wang, and N.-Y. Kim, "High power density AlGaAs/InGaAs/GaAs PHEMTs using an optimised manufacturing process for Ka-band applications," Microelectronic Engineering, vol. 113, pp. 11-19, 2014.

[8] P. Colantonio, F. Giannini, and E. Limiti, High Efficiency RF and Microwave Solid State Power Amplifiers, Department of Electronic Engineering, University of Roma, Tor Vergata, Italy, 2009.

[9] S. Kayali, G. Ponchak, and R. Shaw, GaAs MMIC Reliability Assurance Guideline for Space Applications, Jet Propulsion Laboratory, California Institute of Technology Pasadena, California, Calif, USA, 1996.

[10] W. J. Roesch, "The ROCS workshop and 25 years of compound semiconductor reliability," Microelectronics Reliability, vol. 51, no. 2, pp. 188-194, 2011.

[11] H. J. Siweris, T. Grave, L. Schleicher, H. Riechert, J. E. Muller, and W. Kellner, "A K-band monolithic HEMT medium power amplifier," in Proceedings of the 25th European Microwave Conference, vol. 2, pp. 1105-1109, Bologna, Italy, September 1995.

[12] K. M. Simon, R. M. Wohlert, J. P. Wendler, L. M. Aucoin, and D. W. Vye, "K through Ka-band driver and power amplifiers," in Proceedings of the Microwave and Millimeter-Wave Monolithic Circuits Symposium, Digest of Papers, pp. 29-32, IEEE, June 1996.

[13] Y. G. Kim, J. H. Lee, and C. S. Park, "A PHEMT MMIC broadband power amplifier for LMDS," in Proceedings of the IEEE Radio and Wireless Conference (RAWCON '98), vol. 98, pp. 121124, IEEE, Colorado Springs, Colo, USA, 1998.

[14] P.-C. Huang, Z.-M. Tsai, K.-Y. Lin, and H. Wang, "A 17-35 GHz broadband, high efficiency PHEMT power amplifier using synthesized transformer matching technique," IEEE Transactions on Microwave Theory and Techniques, vol. 60, no. 1, pp. 112-119, 2012.

[15] Y. Sasaki, H. Kurusu, H. Hoshi, T. Hisakaa, and Y. Mitsui, "20-30GHz broadband MMIC power amplifiers with compact flat gain PHEMT cells," in Proceedings of the IEEE MTT-S International Microwave Symposium Digest, vol. 2, pp. 10671070, Phoenix, Ariz, USA, May 2001.

[16] P. Colantonio, F. Giannini, E. Limiti, and V. Teppati, "An approach to harmonic load- and source-pull measurements for high-efficiency PA design," IEEE Transactions on Microwave Theory and Techniques, vol. 52, no. 1, pp. 191-198, 2004.
[17] G. Nikandish, E. Babakrpur, and A. Medi, "A harmonic termination technique for single- and multi-band highefficiency class-F MMIC power amplifiers," IEEE Transactions on Microwave Theory and Techniques, vol. 62, no. 5, pp. 12121220, 2014.

[18] T. K. Quach, P. M. Watson, W. Okamura et al., "Ultrahighefficiency power amplifier for space radar applications," IEEE Journal of Solid-State Circuits, vol. 37, no. 9, pp. 1126-1134, 2002.

[19] AWR, AWR MMIC Flow White Paper, AWR, EI Segundo, Calif, USA, 2012.

[20] ANSYS, ANSYS Icepak Delivers Powerful Technology for Electronics Thermal Management, Application Brief, ANSYS, Canonsburg, Pa, USA, 2011.

[21] S. Scampoli, Staying Cool with ANSYS Icepak: Thermal Management Solution Predicts Air Flow and Heat Transfer in Electronic Designs So Engineers Can Protect Heat-Sensitive Components, ANSYS, Canonsburg, Pa, USA, 2009.

[22] ANSYS, Multiphysics Simulation of a Printed Circuit Board, Application Brief, ANSYS, Canonsburg, Pa, USA, 2014.

[23] T. Zhang, "Ansys second development method and application in transient thermal simulation," in Proceedings of the International Conference on Computer Science and Software Engineering, pp. 643-645, Wuhan, China, December 2008.

[24] J.-S. Lan and M.-L. Wu, "An analytical model for thermal failure analysis of 3D IC packaging," in Proceedings of the 15th International Conference on Thermal, Mechanical and MultiPhysics Simulation and Experiments in Microelectronics and Microsystems (EuroSimE '14), pp. 1-5, Ghent, Belgium, April 2014.

[25] M. Xie, K. C. Toh, and D. Pinjala, "An adaptable compact thermal model for BGA packages," in Proceedings of the 4th Electronics Packaging Technology Conference, pp. 304-311, December 2002.

[26] C. Sohrmann, A. Heinig, M. Dittrich, R. Jancke, and P. Schneider, "Electro-thermal co-design of chip-package-board systems," in Proceedings of the 19th International Workshop on Thermal Investigations of ICs and Systems, Berlin, Germany, September 2013.

[27] S. C. Chen, B. L. Hsiao, F. Chou, K. Yu, H. C. Chou, and C. S. $\mathrm{Wu}$, "An investigation and comparison of 45-degree spread the model and other techniques to extract junction temperature of HBT and PHEMT for reliability life test," in Proceedings of the Reliability of Compound Semiconductors Workshop (ROCS '05), pp. 81-83, 2005. 


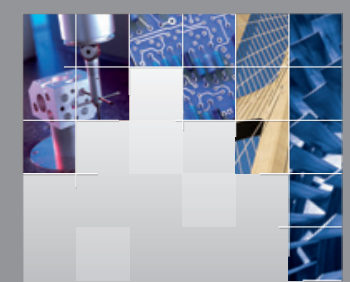

\section{Enfincering}
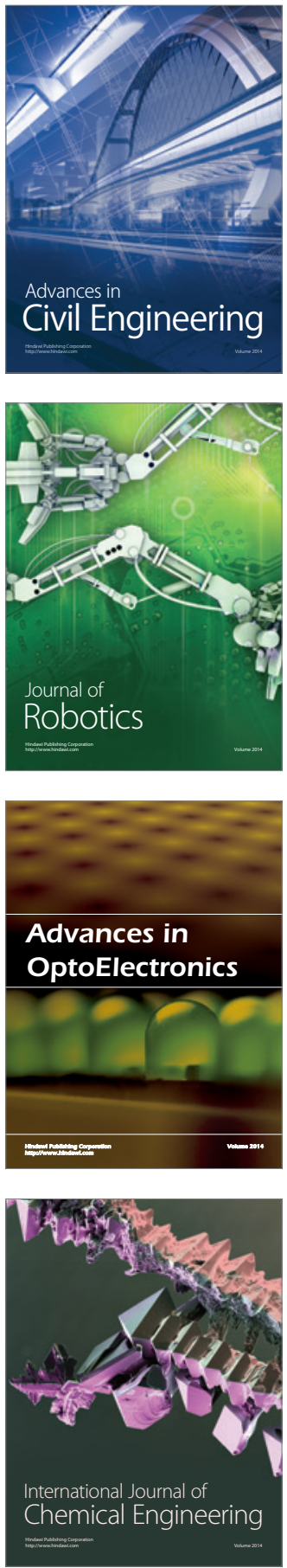

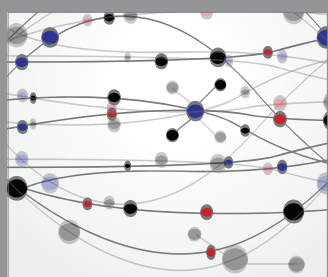

The Scientific World Journal

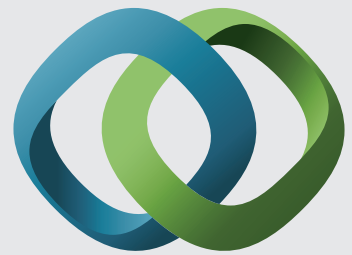

\section{Hindawi}

Submit your manuscripts at

http://www.hindawi.com
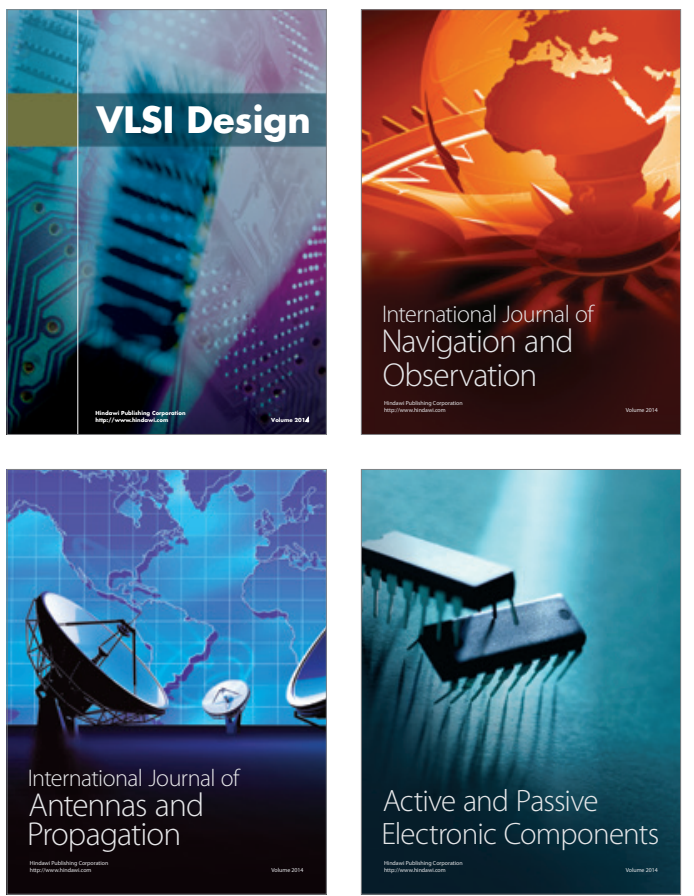
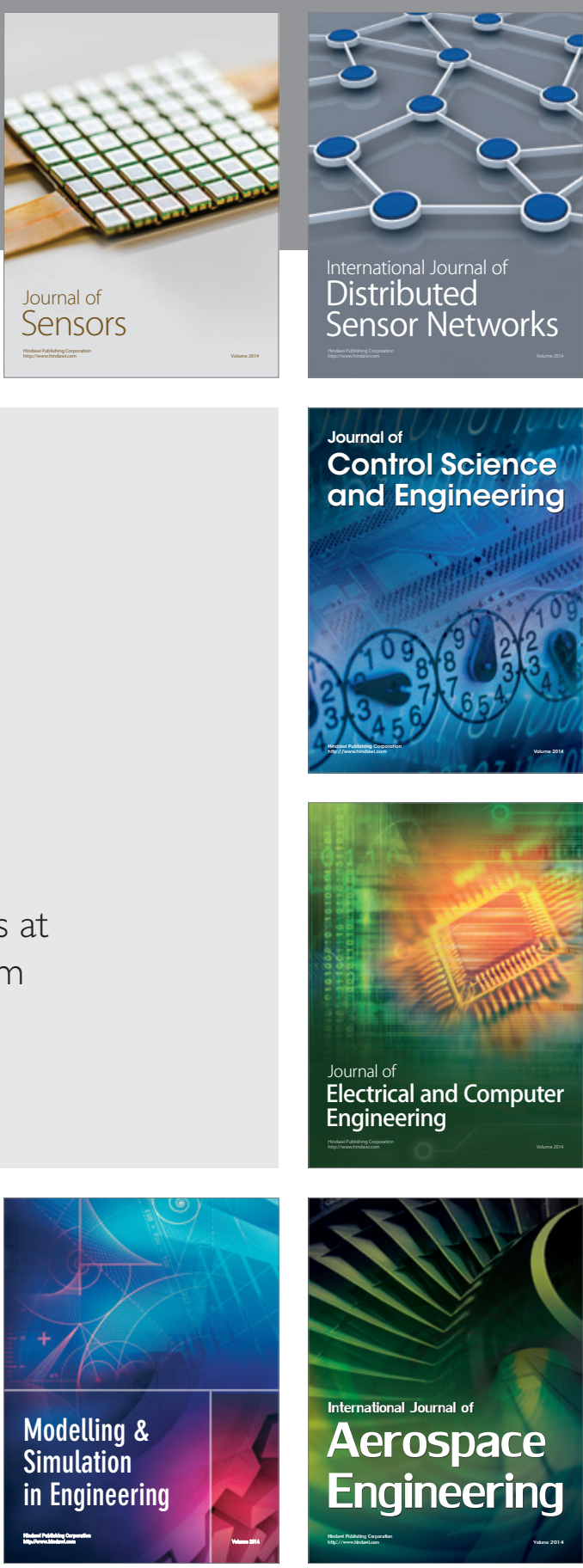

International Journal of

Distributed

Sensor Networks

Journal of

Control Science

and Engineering
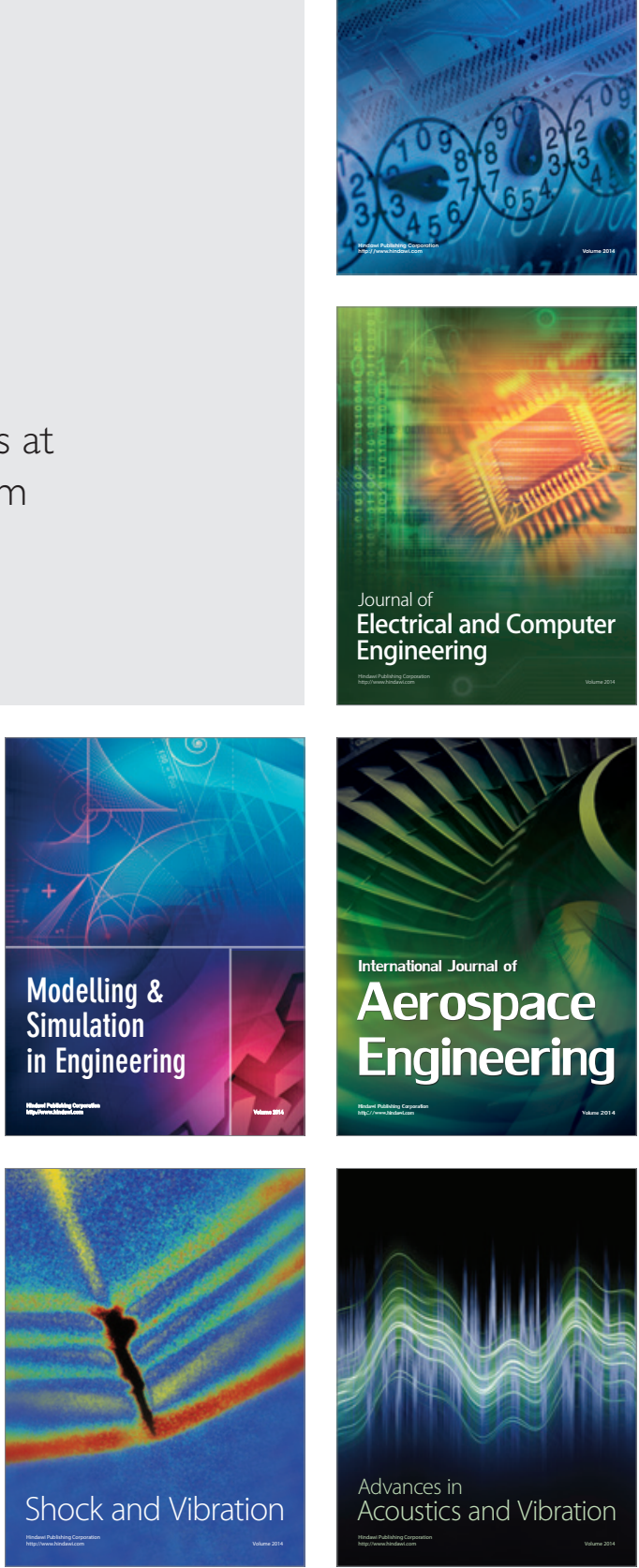